Showa Univ. J. Med. Sci. 4(2), 211 220, December 1992

\title{
Original
}

\section{Treatment for Subtrochanteric Fracture of the Femur}

\author{
Kunihiko Muramatsu, Takashi Yokoyama, Takafumi Kazama, \\ Michio Inoue and Hiromi AKatsu
}

\begin{abstract}
There were 18 cases of subtrochanteric fractures of the femur treated in Kitashinagawa General Hospital during the last five years. These have been examined for fracture type, fixation device, and results. It is very difficult to attain the stability of a fracture which is not only subtrochanteric, but may also be comminuted or intertrochanteric or both. In some cases, an intramedullary nail is the most beneficial for an old patient. However there is always a possibility of complication, such as malunion, especially if it is an unstable fracture. In young and middle age patients, it may be that open reduction with internal fixation (ORIF) is usually better than a blind intramedullary nail. Each device has advantage and disadvantage, therefore in deciding the fixation device, consideration should be given to the exact fracture type, the age and activity of daily living (ADL) of the patient.
\end{abstract}

Key words: subtrochanteric fracture, open reduction/internal fixation, anatomical reduction

\section{Introduction}

Subtrochanteric fractures of the femur occur in the young in traffic or labor accidents ${ }^{1)}$, but many also occur in old age due to a fall. It is generally very difficult to acquire exact anatomical reduction and rigid internal fixation, because comminution, the strong forces exerted by muscles, and dynamic stress concentrated on the hip tend to cause displacement ${ }^{2,3)}$. Consequently, it is often difficult to agree on the most effective treatment. Many methods, such as A-O blade plate, captured hip screw (CHS), and intramedullary nail, are used.

We present here, treatment of subtrochanteric fractures of the femur in our department and describe and discuss the importance of open reduction with internal fixation (ORIF).

\section{Materials and Methods}

The 18 cases treated in our department during the last five years were analyzed for age, sex, cause of injury, fracture type, fixation device, and results. The method of Seinsheimer ${ }^{4}$ was used to classify subtrochanteric fracture of the femur.

\section{Results}

The age and sex of the patients are shown in Fig. 1. The average age was 57.7 years (range, 14 to 83 years). There were 12 men with an average age of 51.4 years and six women with an average of 70.2 years. There were 13 fractures in the right limb and five in

Department of Orthopedic Surgery, Kitashinagawa General Hospital of the Kohno Clinical Medicine Resarch Institute, 3-3-7 Kitashinagawa, Shinagawa-ku, Tokyo 140, Japan. 


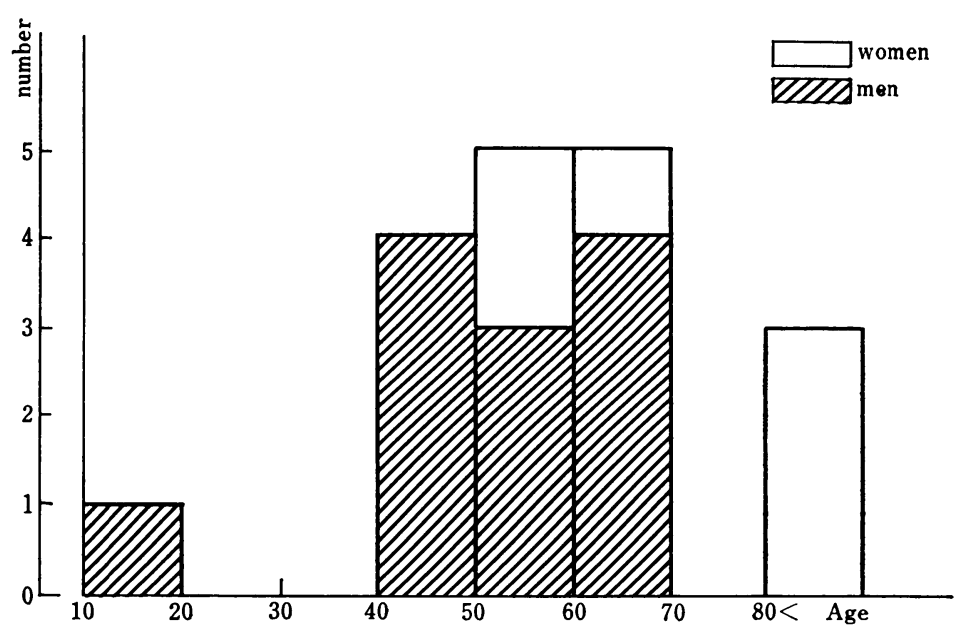

Fig. 1. Distribution of age and sex of the patients are described in this study.

the left. Seven cases were caused in traffic accidents ( 5 of the patients were in motor vehicles, two were pedestrians), five resulted from falls from relatively high places, four were due to stumbling, one was osteogenesis imperfecta and one was due to strain.

As shown in Fig. 2, fractures were classified as defined by Seinsheimer, into type I (1 case), type II A (1 case), type II B (3 cases), type II C (1 case), type III A (3 cases), type III B ( 0 case), type IV ( 3 cases), type V (6 cases). Seventeen of the 18 patients examined in this series were treated surgically. The remaining one, an 83-year-old woman was type I and was treated in traction. She had been treated for sequential apoplexy in a welfare center and had been using a wheelchair for a long time.

Various devices used for ORIF are shown in Table 1. These included: K-A plate (7 cases), CHS ( 5 cases), A-O blade plate (1 case), K-U plate (1 case), Küntscher nail (2 cases), interlocking nail (Grosse \& Kempf) (1 case).

The results of ORIF are shown in Table 2. Sixteen out of 17 fractures treated with ORIF healed without incident $(94.1 \%)$. In the remaining one, treated with a Zickel nail at another hospital, suffered re-fracture soon after extraction, was labeled persistent non-union by roentgenogram findings, and finally failed pseudarthrosis ${ }^{5}$.

Complications included malunion (4 cases; two were varus deformity and two were shortening) and re-fracture $\left.{ }^{6}\right)$ (1 case) after removal of device. According to the assessment for coxarthrosis by The Japanese Orthopaedic Association, the score of clinical findings was 81.3 points and roentgenogram was 3.6 points.

\section{Exhibition}

Case 1: A left subtrochanteric fracture, classified as type V (Fig. 3a), occurred in a 60year-old man who fell from a truck. He was treated with a captured hip screw (Fig. 3b, 3c) and his fracture healed. The final reentgenogram at 16 weeks showed union, but $5 \mathrm{~mm}$ shortening (Fig. 3d).

Case 2: A right subtrochanteric fracture, classified as type V (Fig. 4a), occurred in a 63-year-old man who had been hit by a taxi while riding a scooter. He was treated with a 
Type I 1 case
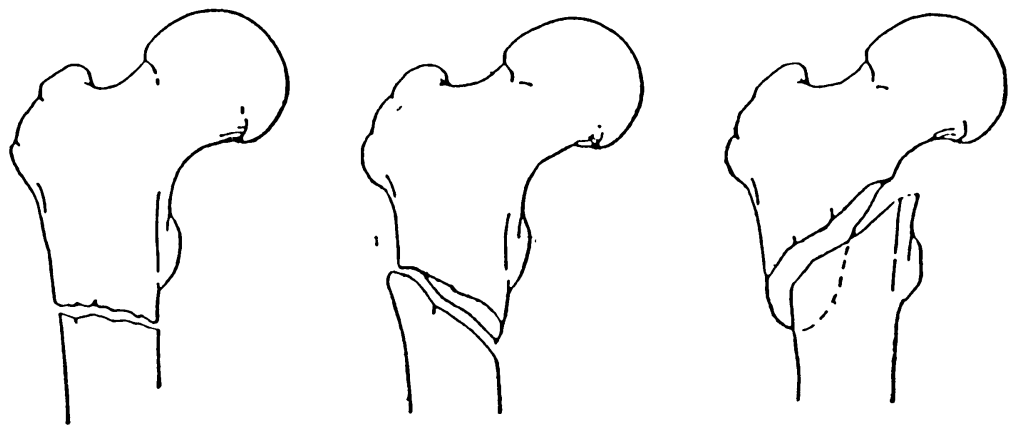

Type II A 1 case

B 3 cases

C 1 case
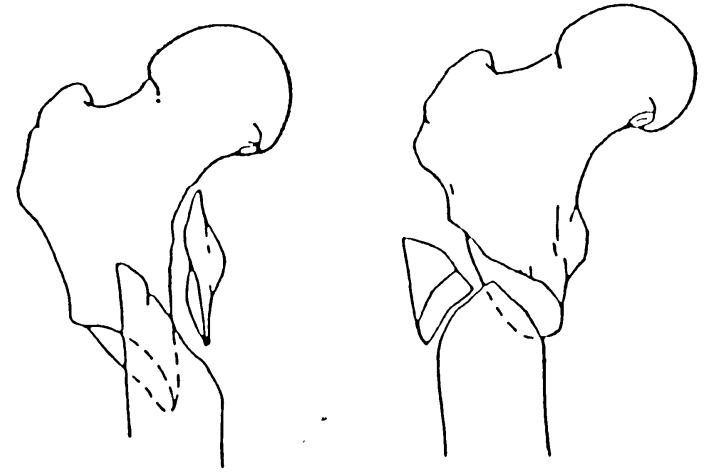

Type III A 3 cases B 0 case

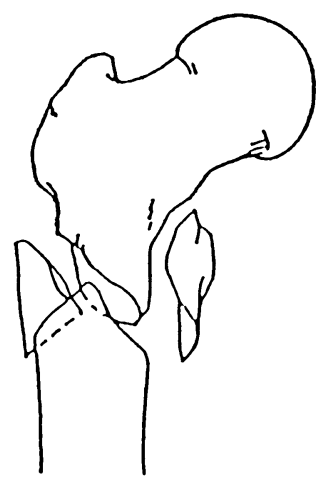

Type IV 3 cases

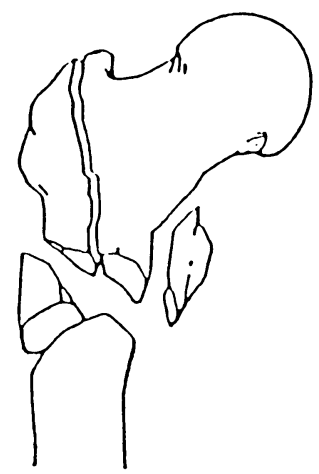

Type V 6 cases

Fig. 2. Subtrochanteric fractures classified as defined by Seinsheimer.

$\mathrm{K}-\mathrm{A}$ plate (Fig. 4b) and his fracture healed uneventfully. At 18 weeks, the final roentgenogram showed union.

Case 3: A left subtrochanteric fracture, classified as type II A occurred in a 14-year-old boy who was affected with osteogenesis imperfecta. He had experienced fractures of his 
Table 1. Types of internal fixation.

\begin{tabular}{lcccccccc}
\hline \multicolumn{1}{c}{ Type } & II A & II B & II C & III A & III B & IV & V & Total \\
A-O blade plate & & 1 & & & & & & 1 \\
K-U plate & & 1 & & & & & & 1 \\
CHS & 1 & & 1 & 1 & & 1 & 2 & 5 \\
K-A plate & & & 1 & & 2 & 3 & 7 \\
Küntscher nail & 1 & 3 & 1 & 3 & 0 & 3 & 6 & 17 \\
Grosse \& Kempf & & 1 & & 1 & & & 1 & 2 \\
Total & 1 & & & & & & \\
\hline
\end{tabular}

Open spaces show no case. The number of patients classified both by the fracture type and the device used for ORIF.

Table 2. Results and complications.

\begin{tabular}{|c|c|c|c|c|c|}
\hline & Union & Pseudarthrosis & Refracture & $\begin{array}{l}\text { Malunion } \\
{\left[\begin{array}{l}\text { Varus deformity } \\
\text { Shortening }\end{array}\right.}\end{array}$ & $\begin{array}{l}\text { Breakage } \\
\text { Bending }\end{array}$ \\
\hline A-O blade plate & 1 & & & & \\
\hline K-U plate & 1 & & & & \\
\hline CHS & 4 & & & {$\left[\begin{array}{l}1 \\
0 \\
1\end{array}\right.$} & \\
\hline $\mathbf{K}-\mathbf{A}$ plate & 7 & & & {$\left[\begin{array}{r}3 \\
2 \\
1\end{array}\right.$} & \\
\hline Küntscher nail & 2 & & & & \\
\hline Grosse \& Kempf & 1 & & & & \\
\hline Zickel & & 1 & 1 & & \\
\hline
\end{tabular}

limbs 22 times since suffering a light epicondylar fracture of the humerus at the age of three. Previously he had suffered a light intracapsular fracture of the femoral neck and had been treated with three cannulated hip screws (Fig. 5a). He was treated with a $\mathrm{K}-\mathrm{A}$ plate (Fig. $5 \mathrm{~b})$ and his fracture healed. At 10 weeks, the roentgenogram showed union.

Case 4: A right subtrochanteric fracture classified as type II B (Fig. 6a), occurred in a 60-year-old man who strained his leg while caring for himself. He was treated with an A-O blade plate (Fig. 6b), and then sent to another hospital where he was treated for sequential apoplexy, unilateral paralysis, and hypertension.

Case 5: A right subtrochanteric fracture classified as type V (Fig. 7a), occurred in a 57year-old man who had been hit by a car while riding a scooter. He was treated with a Küntscher nail (Fig. 7b).

Case 6: A right subtrochanteric fracture classified as type II B occurred in a 55-year-old woman who had jumped from the fourth floor in a suicide attempt. She was first sent to an emergency hospital and treated with a Zickel nail (Fig. 8a). After that she was sent to our hospital for rehabilitation. At 18 months, she suffered re-fracture soon after removal of the 


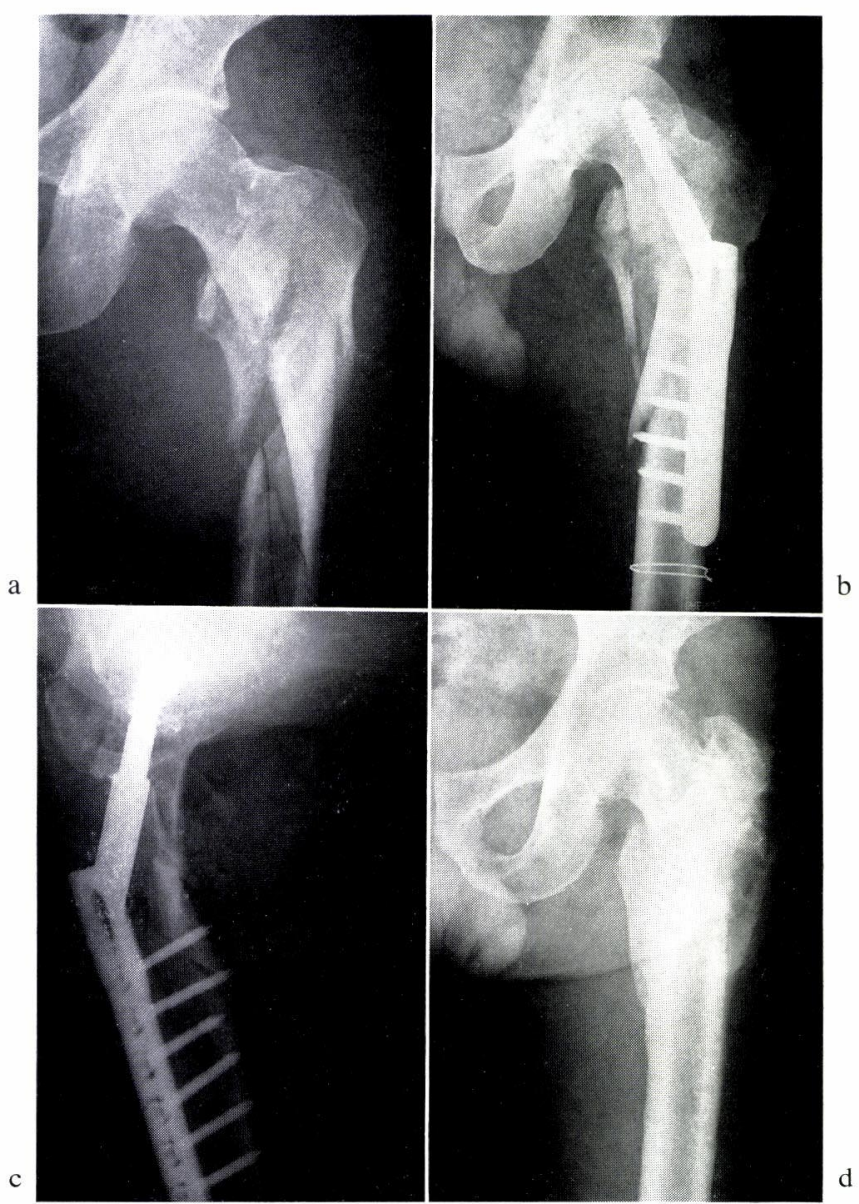

Fig. 3a. The roentgenogram on admission showed a left subtrochanteric fracture, classified as type $\mathrm{V}$.

Fig. 3b. The roentgenogram after operation showed ORIF with a captured hip screw.

Fig. 3c. The roentgenogram after operation showed ORIF with a captured hip screw.

Fig. 3d. The final roentgenogram at 16 weeks showed union, but $5 \mathrm{~mm}$ shortening.

nail (Fig. 8b). She was then treated with a plate and an added bone graft, but had persistent non-union, and finally failed pseudoarthrosis. She was treated with an interlocking nail (Gross \& Kempf) (Fig. 8c) and her fracture healed. At 16 weeks, the last available roentgenogram showed union (Fig. 8d).

\section{Discussion}

It is typical that a subtrochanteric fracture of the femur may not heal because of highly comminuted, displaced femoral cortex and insufficient blood supply. Moreover, a high concentration of stress often occurs in this region, so it is difficult to acquire exact anatomical reduction and rigid internal fixation, comminuted fractures, which often occur in traffic accidents, have a great tendency for failure in ORIF.

The device types are widely divided between nail plate and intramedullary nail. The 


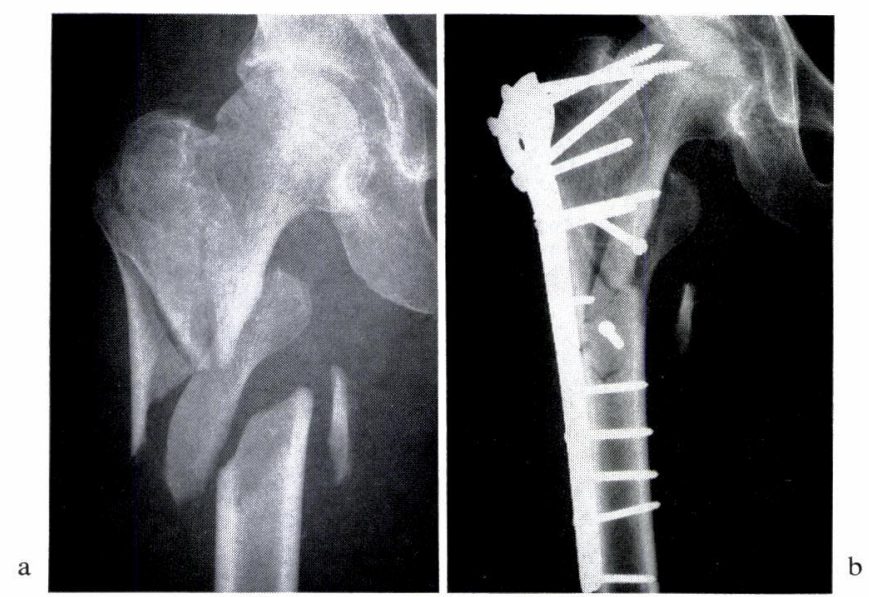

Fig. 4a. The roentgenogram on admission showed a right subtrochanteric fracture, classified as type $\mathrm{V}$.

Fig. 4b. The roentgenogram after operation showed ORIF with a K-A plate.
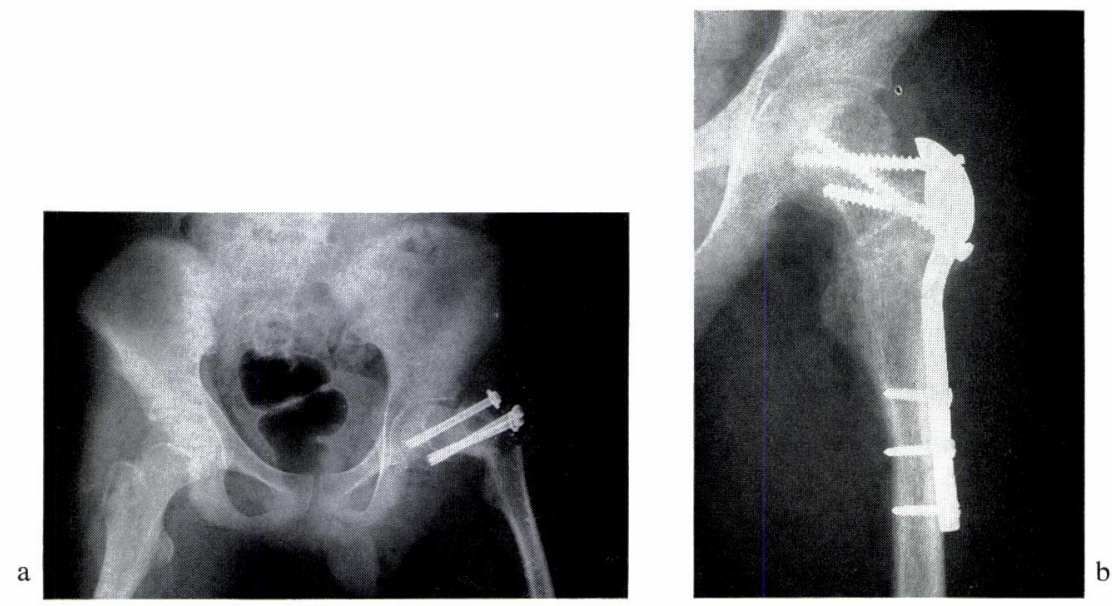

Fig. 5a. The roentgenogram on admission showed a left subtrochanteric fracture, classified as type II A. Previously he had suffered from a light intracapsular fracture of the femoral neck and had been treated with three cannulated hip screws.

Fig. 5b. The roentgenogram after operation showed ORIF with a $\mathrm{K}-\mathrm{A}$ plate.

former include an A-O blade plate and a CHS. The later include a Küntscher nail, an Ender $\operatorname{pin}^{7)}$ and an interlocking nail ${ }^{8)}$.

CHS was used for types II C, III A, IV, and V. The average time to partial weight bearing (PWB) was 10.9 weeks (range, 9 to 12). The mechanism of healing with CHS involves the telescoping of a lag screw into the intramedullary substance to give the fracture compression for healing and produce rigid internal fixation ${ }^{9,10)}$.

CHS is especially effective for intertrochanteric fracture, but is not suitable for unstable types or comminuted fractures. This problem is a matter of ORIF for types III A, IV, and 


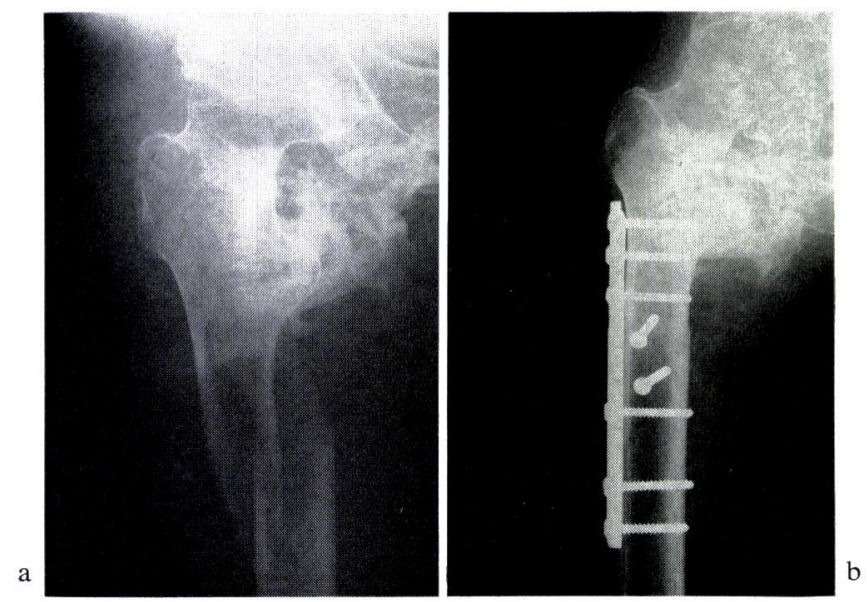

Fig. 6a. The roentgenogram on admission showed a right subtrochanteric fracture, classified as type II B.

Fig. 6b. The roentgenogram after operation showed ORIF with a A-O blade plate.

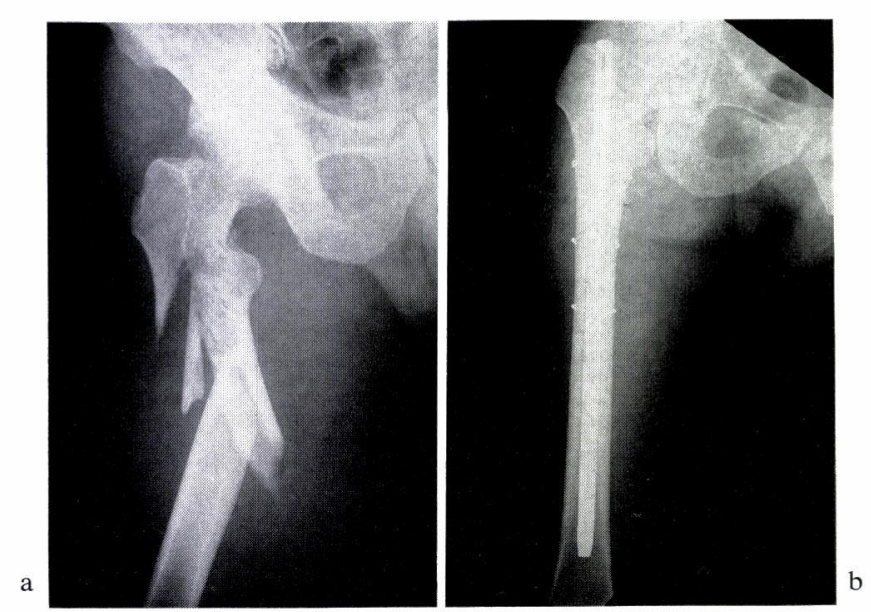

Fig. 7a. The roentgenogram on admission showed a right subtrochanteric fracture, classified as type $\mathrm{V}$.

Fig. 7b. The roentgenogram after operation showed ORIF with a Küntscher nail.

$\mathrm{V}$, because these fractures cannot sustain medial cortical load-bearing, and lie in a position in which a lag screw cannot be inserted and fixed. Anatomical reduction and rigid internal fixation cannot be established by only a lag screw. In some cases they also need wiring, a long tube plate, or external fixation for some period of weeks. Shortening may often arise and consequently it is necessary to be careful of PWB.

A K-A plate was used for types II A, III B, IV, and V. The average period to PWB was 9.7 weeks (range, 8 to 12). Some cases included unstable types, such as comminuted fractures, which necessifated wiring. Varus deformity may often arise from types IV and V. This also demands strict attention to PWB. 


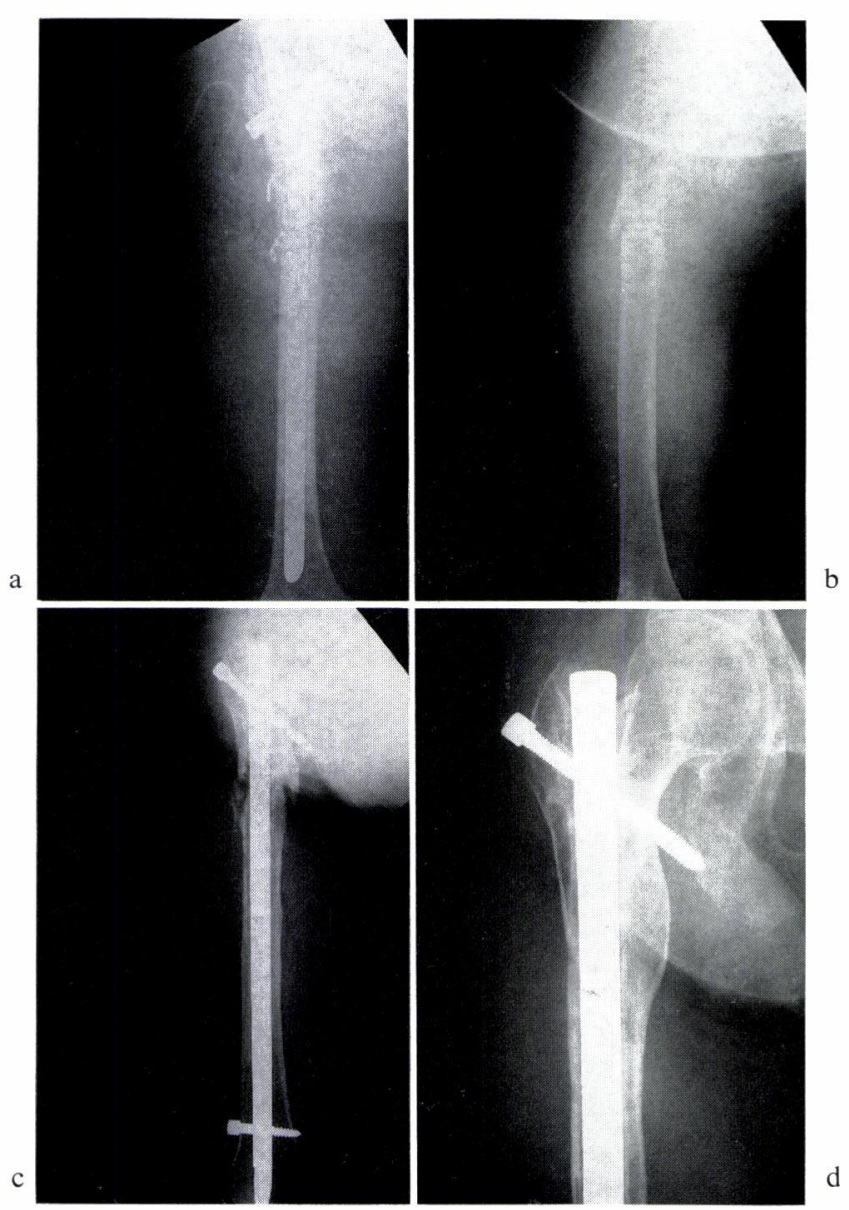

Fig. 8a. The roentgenogram on admission showed a right subtrochanteric fracture, classified as type II B. She was first sent to an emergency hospital and treated with a Zickel nail.

Fig. 8b. The roentgenogram soon after extraction of the nail at 18 months showed re-fracture.

Fig. 8c. The roentgenogram after the final operation showed ORIF with an interlocking nail.

Fig. 8d. The last available roentgenogram at 16 weeks after the final operation showed union.

An A-O blade plate was used for type II B. This is adaptable for types II and III B in which medial cortical support is adequate ${ }^{11)}$. If the internal fixation over-depends on the strength of the plate for types III A, IV, and V, in which medial cortical support is inadequate $^{9)}$, breakage, bending or re-fracture may occur ${ }^{12)}$.

A Küntscher nail was used for types III A and V. The average time to PWB was 4.5 weeks (range, 3 to 6). The Küntscher nail is adaptable to a transverse fracture such as type II A, or short spiral fracture such as types II B, II $\mathrm{C}^{11,13)}$, but long spiral or unstable fractures, or osteoporosis, together with third fragments could develop problems. If PWB occurs too early, there may be a high risk of prolapse with the Küntscher nail. We should thus be extra careful of PWB. 
An interlocking nail was used for type II B. The patient had been treated with a Zickel nail in another hospital, but pseudarthrosis failed. At 16 weeks, after the final operation with an interlocking nail, her fracture healed and the roentgenogram showed union. This is adaptable for types II A, II B and III B in which greater or lesser trochanter is adequate ${ }^{11)}$, but the Küntscher nail should be seriously considered.

The intramedullary nail is reported to be excellent, and has produced good results in recent years. However, there is still room for discussion about treatment with either the intramedullary nail or a nail plate. The intramedullary nail is especially very difficult to use for treatment of old patients who are in poor condition or osteoporotic. It is possible to injure the cortex or cause malunion if reaming is done in the region where the fracture line extends, and the device should be inserted. The Ender pin is excellent because of the possibility of closed treatment and low risk for old patients, but it is difficult to produce rigid internal fixation with flexible pinning. It is also possible to experience malunion as with a nail plate ${ }^{1,14)}$. Varus deformity is also possible. The interlocking nail can not be fully used to its potential if the fracture line extends intertrochanteric between the greater and lesser trochanters.

The aim of treatment with a nail plate is anatomical reduction. However, the disadvantages are: 1) The periosteum is easily injured by open reduction and may result in delayed appearance of callus. 2) It requires time to operate for open reduction, and bleeding is always possible. 3) Breakage or re-fracture may occur if the nail plate is stressed beyond its strength.

Therefore, every device has its advantages and disadvantages and anatomical reduction without malunion should be considered, especially for younger or middle age patients ${ }^{(1)}$. At the operation, the periosteum must be preserved as much as possible and sometimes wiring is useful for fixation of fragments. If the fixation reveals instability or causes bone defect, it may require the addition of bone grafts or indication of non-weight bearing (NWB) or external fixation for some period ${ }^{11,15)}$.

Judging from these reports and the present results, it is reasonable to propose that all devices should be considered based on the patients' fracture type, age, ADL, and any other pertinent factors. Finally, it is necessary that we master different types of operative techniques so the method which is most beneficial can be selected.

\section{Conclusion}

1) Subtrochanteric fractures of the femur were treated for 18 patients in our department in the last five years.

2) Great care should be taken in the choice of internal fixation and post-operative treatment for unstable or comminuted fractures that are not only subtrochanteric but also intertrochanteric.

3) Special care is required for anatomical reduction for young or middle age patients and avoidance of malunion.

4) In reductioning, periosteum must be preserved as much as possible, moreover according to circumstances, it may be necessary to add bone graft, to indicate NWB, and to use external fixation for some period.

5) We had better choose internal fixation only after consideration of the fracture type, age, ADL, and other factors. Since each device has advantages and disadvantages, we should not be limited to only one method. 


\section{Acknowledgment}

We thank Dr. K. Asano for his useful advice.

\section{References}

1) Watson HK, Campbell RD and Wade PA: Classification, treatment and complications of the adult subtrochanteric fracture. J Trauma, 4: 457-480 (1964)

2) Koch JC: The laws of bone architecture. Am J Anat, 21: 177-298 (1917)

3) Saito $\mathrm{H}$ : AO condylar plate fixation for subtrochanteric fractures of the femur. Joint, 2: 28-34 (1987) (in Japanese)

4) Seinsheimer F: Subtrochanteric fractures of the femur. J Bone Joint Surg [Am], 60: 300-306 (1978)

5) Ovadia DN and Chess JL: Intraoperative and postoperative subtrochanteric fracture of the femur associated with removal of the Zickel nail. J Bone Joint Surg [Am], 70: 239-243 (1988)

6) Fielding JW and Magliato HJ: Subtrochanteric fractures. Surg Gynecol Obstet, 122: 555-560 (1966)

7) Ender HG and Schneider H: Subtrochantere bruche des Oberschenkels: Behandlung mit Federnageln. Aktuel Chir, 9: 359-372 (1974) (with English summary)

8) Kempf I, Grosse A and Beck B: Closed locked intramedullary nailing. J Bone Joint Surg [Am], 67: 709-720 (1985)

9) Ruff ME and Lubbers LM: Treatment of subtrochanteric fractures with a sliding screw-plate device. J Trauma, 26: 75-80 (1986)

10) Schatzker J and Waddell JP: Subtrochanteric fractures of the femur. Orthop Clin North Am, 11: 539-544 (1980)

11) Trafton PG: Subtrochanteric-intertrochanteric femoral fractures. Orthop Clin North Am, 18: 59-71 (1987)

12) Waddell JP: Subtrochanteric fractures of the femur a review of 130 patients. J Trauma, 19: 582592 (1979)

13) Rockwood CA: Subtrochanteric fractures. In: Fractures in Adults, 2, J.B. Lippincott Company, Philadelphia, pp 1277-1287 (1984)

14) Dobozi WR: Flexible intramedullary nailing of subtrochanteric fractures of the femur. Clin Orthop Relat Res, 212: 68-78 (1985)

15) Velasco RU and Comfort TH: Analysis of treatment problems in subtrochanteric fractures of the femur. J Trauma, 18: 513-523 (1978)

[Received September 28, 1992: Accepted October 16, 1992] 